\title{
Osthole decreases renal ischemia-reperfusion injury by suppressing JAK2/STAT3 signaling activation
}

\author{
LIN-NA LUO ${ }^{1,2^{*}}$, DE QIONG XIE ${ }^{1,2^{*}}$, XIAO GANG ZHANG ${ }^{2}$ and RONG JIANG ${ }^{3}$ \\ ${ }^{1}$ Department of Intensive Care, West China Fourth Hospital of Sichuan University, Chengdu, Sichuan 610041; \\ ${ }^{2}$ Department of Nephrology, The First Affiliated Hospital of Chongqing Medical University, Chongqing 400042; \\ ${ }^{3}$ Department of Internal Medicine, University of Electronic Science and Technology, \\ Sichuan Academy of Sciences \& Sichuan Provincial People's Hospital, Chengdu, Sichuan 610072, P.R. China
}

Received January 26, 2016; Accepted February 18, 2016

DOI: $10.3892 /$ etm.2016.3603

\begin{abstract}
Renal ischemia-reperfusion (I/R) injury is a major cause of acute kidney injury. The pathogenetic mechanisms underlying renal I/R injury involve inflammation, oxidative stress and apoptosis. Osthole is a coumarin derivative that exhibits potential anti-inflammatory activity. The aim of the present study was to investigate the effect of osthole in renal I/R injury and its underlying mechanism. Renal I/R injury was induced by clamping the left renal artery for $45 \mathrm{~min}$ followed by $24 \mathrm{~h}$ reperfusion with the contralateral nephrectomy. A total of 70 rats were randomly assigned to seven groups $(n=10$ per group): Sham; IRI; and osthole $(0,5,10,20$ and $40 \mathrm{mg} / \mathrm{kg})$ groups. Rats were administered intraperitoneally with osthole $45 \mathrm{~min}$ prior to renal ischemia. Serum and renal tissue were harvested $24 \mathrm{~h}$ after reperfusion. Renal function and histological changes were assessed. In addition, the mRNA and protein expression of tumor necrosis factor- $\alpha$ (TNF- $\alpha)$, interleukin- 8 (IL-8) and interleukin-6 (IL-6) in renal tissue and serum were evaluated using quantitative polymerase chain reaction and ELISA assays, respectively. The protein expression levels of p65, p-p65, janus kinase 2 (JAK2), p-JAK2, signal transducer and activator of transcription 3 (STAT3) and p-STAT3 were measured using western blot analysis. The results indicate that osthole pretreatment was able to significantly attenuate the renal dysfunction in a dose-dependent manner, histological changes and the expression of TNF- $\alpha$, IL-8, IL-6, p-JAK2, p-STAT3 and p-p65 induced by renal I/R injury. However,
\end{abstract}

Correspondence to: Rong Jiang, Department of Internal Medicine, University of Electronic Science and Technology, Sichuan Academy of Sciences \& Sichuan Provincial People's Hospital, 32 West Second Street, Chengdu, Sichuan 610072, P.R. China

E-mail: 1285396756@qq.com

*Contributed equally

Key words: osthole, renal, ischemia reperfusion injury, inflammation, janus kinase 2/signal transducer and activator of transcription 3 neither osthole or I/R injury affected the expression p65, JAK2 and STAT3. Osthole pretreatment is able to reduce renal $\mathrm{I} / \mathrm{R}$ injury by abrogating inflammation and the mechanism is partially involved in suppressing JAK2/STAT3 activation. Thus, osthole may be a novel practical strategy for the mitigation of renal I/R injury.

\section{Introduction}

Renal ischemia is commonly observed in patients with cardiovascular surgery, trauma, shock, burn and those that have undergone organ transplantation $(1,2)$. Reperfusion of ischemic tissue is associated with increased production of oxygen radicals, subsequently leading to endothelial barrier dysfunction and tissue injury (3).

Ischemia-reperfusion ( $\mathrm{I} / \mathrm{R})$ injury may result in a molecular and cellular inflammatory response within the kidney, which can induce the activation of the inflammation associated transcription factor nuclear factor $-\kappa B$ $(\mathrm{NF}-\kappa \mathrm{B})$, which is crucially involved in the pathogenesis of I/R injury $(4,5)$. Notably, the increased activation of $N F-\kappa B$ in the I/R-challenged kidney further contributes to kidney tissue damage, frequently causing systemic a inflammatory response and subsequent leading to acute kidney failure (5). Therefore, suppressing NF- $\mathrm{NB}$ mediated inflammation may be an effective measure to attenuate renal ischemia reperfusion injury.

The Janus kinase/signal transducer 2 and activator of transcription 3 (JAK2/STAT3) pathway is a classical signaling pathway that transduces cellular signals from the plasma membrane to the nucleus, and has an important role in regulating NF- $\mathrm{NB}$-mediated inflammation (6).

Osthole is a naturally-derived component of coumarin, which has been widely used clinically owing to its multiple biochemical and pharmacological effects (7). Furthermore, previous studies have demonstrated that osthole has a protective effect in cerebral intestinal I/R injury by exerting anti-inflammatory effects $(7,8)$.

However, the role and potential molecular mechanisms of osthole in modulation of I/R-induced inflammatory response in the kidney remain unclear. Therefore, the present study aimed to investigate the effects and potential mechanisms of 
osthole in modulation of I/R-induced inflammatory response in rats kidney.

\section{Materials and methods}

Ethical approval. The experimental protocol was approved by the Institutional Animal Care and Use Committee at the First Affiliated Hospital of Chongqing Medical University (Chongqing, China).

Drug. Osthole (purity, >98\%) was purchased from the National Institute for Food and Drug Control (Beijing, China). Osthole was dissolved in a 1:9 (v/v) mixture of Tween 80 (Google Biological Technology Co., Ltd., Wuhan, China) and $0.9 \%$ sodium chloride (Google Biological Technology Co., Ltd.).

Animals. Male Sprague-Dawley rats (weight, 180-240 g) were purchased from Hua Fukang Experimental Animal Center (Beijing, China). The rats were housed in a specific pathogen-free facility and fed with laboratory chow and ad libitum water. After a minimum seven days of acclimation, the rats were randomly allocated into seven groups $(n=10$ per group): i) Sham-operated group (sham), in which the rats were subjected to identical surgical procedure without occlusion of renal pedicles; ii) I/R-vehicle group (IRI), in which the rats were subjected to renal ischemia for $45 \mathrm{~min}$; and I/R-osthole group (osthole), in which the rats were administered osthole $(0$, $5,10,20$ or $40 \mathrm{mg} / \mathrm{kg}$, intravenously) $45 \mathrm{~min}$ prior to I/R induction. The dosage of osthole was based on a previous study (9).

$I / R$ induction in the kidneys. The rats were first anesthetized with an intraperitoneal injection of $1 \%$ sodium pentobarbital solution (65 mg/kg; Google Biological Technology Co., Ltd.) and a rectal probe was inserted to monitor body temperature, which was maintained at $37 \pm 1^{\circ} \mathrm{C}$ using a heating blanket. A midline laparotomy was performed and the abdominal cavity was fully exposed.

Bilateral renal pedicles were carefully isolated without damaging the ureter and clamped by non-traumatic microvascular clamps to effect complete cessation of renal arterial blood flow. After $45 \mathrm{~min}$, the clamps were removed to allow return of blood flow to the kidneys. Successful ischemia or reperfusion was judged by observing the change in tissue color from red to dark blue or from dark blue to bright red respectively, the contralateral kidney was removed. Middle abdominal incisions were closed in two layers and covered with antibiotic ointment when the operation finished. The animals were allowed to recover from anesthesia, remaining $24 \mathrm{~h}$ in a controlled-environment room with food and water freely available. Rats in the sham group underwent laparotomy without performing renal ischemia, as a control population. The rats were sacrificed with an intraperitoneal injection of $1 \%$ sodium pentobarbital solution (120 mg/kg body weight) $24 \mathrm{~h}$ after reperfusion, and the kidneys were harvested for further analysis.

Assessment of renal function. Serum creatinine (Cr) and blood urea nitrogen (BUN) were used as indicators of impaired renal function. Blood samples were obtained from the inferior vena cava $24 \mathrm{~h}$ after reperfusion and were placed in the refrigerator at $4^{\circ} \mathrm{C}$ for $20 \mathrm{~min}$ and centrifuged $(6,000 \mathrm{x}$ g for $3 \mathrm{~min})$ to separate the serum. The biochemical parameters (BUN and Cr levels) were analyzed photometrically with an autoanalyzer (AU5800; Beckman Coulter, Inc, Brea, CA, USA) in the core laboratory of the First Affiliated Hospital of Chongqing Medical University for assessment of renal function.

Histological analysis. Renal samples were fixed in formalin and then embedded in paraffin, and renal sections were next prepared and subjected to hematoxylin and eosin (HE) staining, as reported previously (10). The histopathological changes in the cortex and medulla were evaluated by a pathologist in a blinded fashion using a five-point quantitative scale according to the degree of tubular necrosis, hemorrhage and cast formation, as follows: $0,<10 \% ; 1,10-25 \% ; 2,25-50 \% ; 3,50-75 \%$; and $4,75-100 \%$ (11).

Western blot analysis. The renal tissue was lysed in ice-cold radioimmunoprecipitation assay buffer [50 mM Tris $(\mathrm{pH} 7.4)$, $150 \mathrm{mM} \mathrm{NaCl}, 1 \%$ Triton, $0.5 \%$ deoxycholate, $0.1 \%$ SDS, $1 \mathrm{mM}$ EDTA, $10 \mathrm{mM} \mathrm{NaF}$, and $0.1 \mathrm{mM}$ phenylmethylsulfonyl fluoride] to obtain the total proteins. The protein concentration was detected using a bicinchoninic acid protein assay (Beyotime Institute of Biotechnology, Shanghai, China). Equal amounts of protein samples $(50 \mu \mathrm{g}$ protein/lane) were separated by 10-12\% SDS-PAGE and transferred onto polyvinylidene difluoride membranes. The non-specific antibodies were blocked with $5 \%$ non-fat dried milk in phosphate-buffered saline for $2 \mathrm{~h}$ at room temperature. The membranes were then incubated overnight at $4^{\circ} \mathrm{C}$ with primary antibodies directed against JAK2 (cat. no. ab39636; 1:1,000; Abcam, Cambridge, UK), p-JAK2 (cat. no. ab32101; 1:500, Abcam), STAT3 (cat. no. ab68153; 1:1,000; Abcam), p-STAT3 (cat.no. ab76351; 1:500; Abcam), p65 (cat. no. ab16502; 1:1,000; Abcam) and p-p65 (cat. no. ab86299; 1:500; Abcam). The membranes were then washed with Tris-buffered saline with Tween 20 three times and further incubated with horseradish peroxidase conjugated secondary antibody (1:3,000; Jackson ImmunoResearch Laboratories, Inc, West Grove, PA, USA) for $1 \mathrm{~h}$ at room temperature. Following washing, the membranes were processed using an electrochemiluminescence reagent (GE Healthcare Bio-Sciences, Pittsburg, PA, USA) and the light emission was captured on X-ray film. The signals were visualized by chemiluminescent horseradish peroxidase substrate and then subjected to a densitometric analysis and normalized to $\beta$-actin (1:3,000; Abmart Co, Ltd., Shanghai, China).

Reverse transcription-quantitative polymerase chain reaction $(R T-q P C R)$ analysis. Total RNA was isolated from renal tissue using RNAiso Plus reagent (Takara Bio, Inc, Otsu, Japan). RNA was treated with RNase-free DNase I to remove gDNA. Absorbances at 260 and $280 \mathrm{~nm}$ were measured for RNA quantification and quality control. All RNA samples exhibited high quality RNA and were subsequently reverse transcribed to cDNA using a PrimeScript ${ }^{\mathrm{TM}}$ RT reagent kit (Perfect Real Time; Takara Bio, Inc.) according to the manufacturer's instructions. Subsequently, qPCR was conducted to determine the levels of mRNA expression using an ABI Prism 7000 sequence detection system (Applied Biosystems; 
Table I. Primers used for quantitative polymerase chain reaction analysis.

\begin{tabular}{lll}
\hline Gene & \multicolumn{1}{c}{ Sense strand sequence } & Anti-sense strand sequence \\
\hline TNF- $\alpha$ & CTGAACTTCGGGGTGATCGG & GGCTTGTCACTCGAATTTTGAGA \\
IL-6 & AGCTTCCTTGTGCAAGTGTCT & GACAGCCCAGGTCAAAGGTT \\
IL- 8 & CTGCAAGAGACTTCCATCCAG & AGTGGTATAGACAGGTCTGTTGG \\
\hline
\end{tabular}

TNF- $\alpha$, tumor necrosis factor- $\alpha$; IL, interleukin.

Thermo Fisher Scientific, Inc., Waltham, MA, USA) in triplicate in 96-well plates in a final volume of $20 \mu$ l under standard conditions. qPCR was conducted on cDNA samples using the SYBR Green method with SYBR ${ }^{\circledR}$ Premix Ex Taq ${ }^{\mathrm{TM}}$ (Tli RNaseH Plus; Takara Bio, Inc.). Reaction mixtures contained $10 \mu 12 X$ SYBR Green mastermix, $1 \mu \mathrm{l}(6 \mu \mathrm{M})$ forward primer, $1 \mu \mathrm{l}(6 \mu \mathrm{M})$ reverse primer, $6 \mu \mathrm{l}$ water and $2 \mu \mathrm{l}(5 \mathrm{ng} / \mu \mathrm{l}) \mathrm{cDNA}$. qPCR was performed as follows: Initial denaturation at $95^{\circ} \mathrm{C}$ for $30 \mathrm{sec}$ for activation of AmpliTaq Cold DNA polymerase (Applied Biosystems; Thermo Fisher Scientific, Inc.), followed by 40 cycles of denaturation at $95^{\circ} \mathrm{C}$ for $5 \mathrm{sec}$, annealing at $60^{\circ} \mathrm{C}$ for $30 \mathrm{sec}$, and extension at $95^{\circ} \mathrm{C}$ for $15 \mathrm{sec}$. Forward and reverse primer sequences are listed in Table I and were synthesized by Takara Bio, Inc. To normalize each sample, a control gene ( $\beta$-actin) was used, and the arbitrary intensity threshold of amplification was computed. The $2^{-\Delta \Delta \mathrm{Cq}}$ method was used to calculate the relative expression of each target gene, as described previously (12), and analyzed using SPSS 12.0 software (SPSS, Inc, Chicago, IL, USA).

ELISA analysis. Levels of the inflammatory mediators TNF- $\alpha$ (Bosde Biotechnology, Wuhan, China; QN-PS1726), IL-6 (Bosde Biotechnology; EK0526) and IL-8 (Bosde Biotechnology; BA-3381) in the serum were quantified using specific ELISA kits for mice according to the manufacturer's instructions (BioSource International, Inc., Camarillo, CA, USA).

Statistical analysis. All results are expressed as the mean \pm standard error of the mean of three independent experiments. Differences among groups were assessed using a one way analysis of variance. Least significant difference $t$ tests were used when a single control group was compared with all other groups. All statistical analyses were conducted using SPSS 13.0 software (SPSS, Inc.). $\mathrm{P}<0.05$ was considered to indicate a statistically significant difference.

\section{Results}

Osthole pretreatment decreased renal dysfunction after $I / R$-induced renal injury. As shown in Fig. 1A and B, compared with the sham group, rats in IRI group showed a significant increase in the levels of $\mathrm{Cr}$ and BUN $(\mathrm{P}<0.001)$. However, osthole pretreatment significantly decreased the levels of $\mathrm{Cr}$ and BUN induced by renal I/R injury in a dose-dependent manner. The optimal concentration of osthole for the protective effect was $40 \mathrm{mg} / \mathrm{kg}(\mathrm{P}<0.001)$.
Osthole pretreatment decreased pathological changes of the kidney after renal I/R. Compared with the sham group (Fig. 2A), the rats in IRI group showed significant pathological changes, including widespread degeneration of the tubular architecture, tubular dilation, tubular cell swelling, cellular vacuolization, tubular cell necrosis and inflammatory cell infiltration (Fig. 2B). However, pretreatment with osthole resulted in reduced pathological change in kidney as compared with the IRI group (Fig. 2C). The histopathological score of the rats' renal in all groups are presented in Fig. 2D. The scores of IRI group were higher compared with the sham and osthole groups $(\mathrm{P}<0.001)$.

Osthole pretreatment can reduce the expression of proinflammatory cytokine expression after renal IRI. To determine whether osthole pretreatment can interfere with I/R-induced inflammatory response in the kidney, with respect to production of inflammation-relevant cytokines TNF- $\alpha$, IL- 6 and IL- 8 , the renal tissue obtained from each group of rats were assessed for TNF- $\alpha$, IL- 6 and IL- 8 mRNA expression level. As shown in Fig. 3, the mRNA expression levels of TNF- $\alpha$, IL- 6 and IL- 8 were significantly upregulated in I/R-damaged kidneys. Notably, the elevated levels of kidney TNF- $\alpha$, IL- 6 and IL- 8 mRNA expression were effectively reduced by preconditioning with osthole.

Osthole pretreatment can reduce the secretion of proinflammatory cytokines after renal IRI. To further demonstrate the effect of osthole pretreatment in interfering with I/R-induced inflammatory response in the IRI, the serum obtained from each group of rats were assessed for TNF- $\alpha$, IL-6 and IL-8 secretion level. As shown in Fig. 4, the secretion level of TNF- $\alpha$, IL- 6 and IL- 8 were significantly upregulated in the I/R-challenged serum. Notably, the elevated levels of serum TNF- $\alpha$, IL-6 and IL-8 were effectively reduced by preconditioning with osthole.

Osthole pretreatment can attenuate $N F-\kappa B$ activation after renal $I / R$ injury. $\mathrm{NF}-\kappa \mathrm{B}$ is crucially involved in the inflammatory response in renal $\mathrm{I} / \mathrm{R}$, and its activation is dependent p65 activation $(4,5)$. As shown Fig. 5, neither I/R injury nor osthole pretreatment significantly affected p65 expression. However, the quantity of activated p65 (p-p65) noted in rats after I/R injury was significantly higher compared with the sham control rats (Fig. 5A and B). By contrast, pretreatment with osthole can significantly reduce p65 activation, as manifested by the lower levels of p-p65 detected in osthole group 
A



B

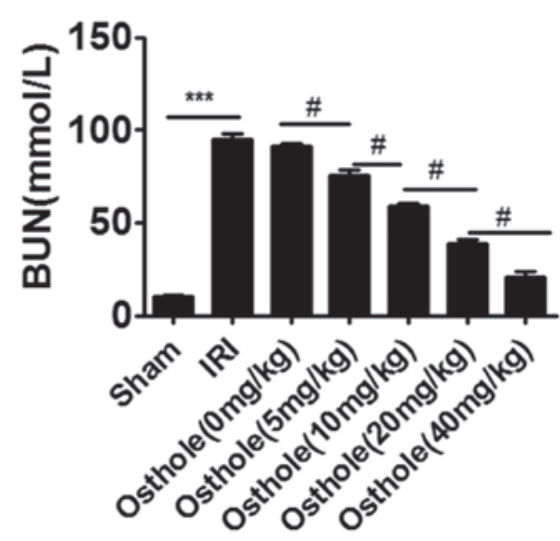

Figure 1. Effects of osthole pretreatment on alterations of renal function following renal ischemia/reperfusion (I/R)-induced injury. (A) Serum $\mathrm{Cr}$ and (B) BUN levels were evaluated to assess the renoprotective effect of against renal I/R osthole pretreatment injury in the sham, IRI and osthole groups. Data are presented as the mean \pm standard error of the mean $(n=10)$ ${ }^{* * *} \mathrm{P}<0.001$ vs. sham; ${ }^{\#} \mathrm{P}<0.005$ vs. osthole. $\mathrm{Cr}$, creatinine; IRI, ischemia/ reperfusion vehicle group; BUN, blood urea nitrogen.

rats as compared with that of the IRI group rats. Collectively, the present results suggest that osthole pretreatment can attenuate inflammatory response in renal ischemia reperfusion injury by reducing NF-kB activation (Fig. 5A and B).

Osthole pretreatment suppressed JAK2/STAT3 activation after renal $I / R$. In order to further investigate the mechanism underlying the osthole-mediated decreasing renal I/R injury, the activity of JAK2/STAT3 signaling was selectively analyzed in renal I/R injury. As shown in Fig. 6A and B, neither $\mathrm{I} / \mathrm{R}$ insult nor osthole pretreatment have influence on the expression of JAK2. However, IRI can increase the activation of JAK2, as indicated by the increased levels of p-JAK2 in the IRI group compared with the sham group. Furthermore, osthole pretreatment can decrease the effect of IRI, inducing the activation of JAK2, as manifested lower levels of p-JAK2 in osthole group compared with the IRI group (Fig. 6A and B). Since JAK2 activation may provide signals to STAT3, we next evaluated the expression of STAT3. The results showed that I/R injury and osthole have no influence on the expression of STAT3 (Fig. 6C and D); however, I/R injury can increased the expression of p-STAT3, while osthole pretreatment can decrease the expression of p-STAT3 induced by I/R injury. Collectively, the present data indicated that osthole pretreatment can decrease JAK2/STAT3 signaling following renal I/R injury.
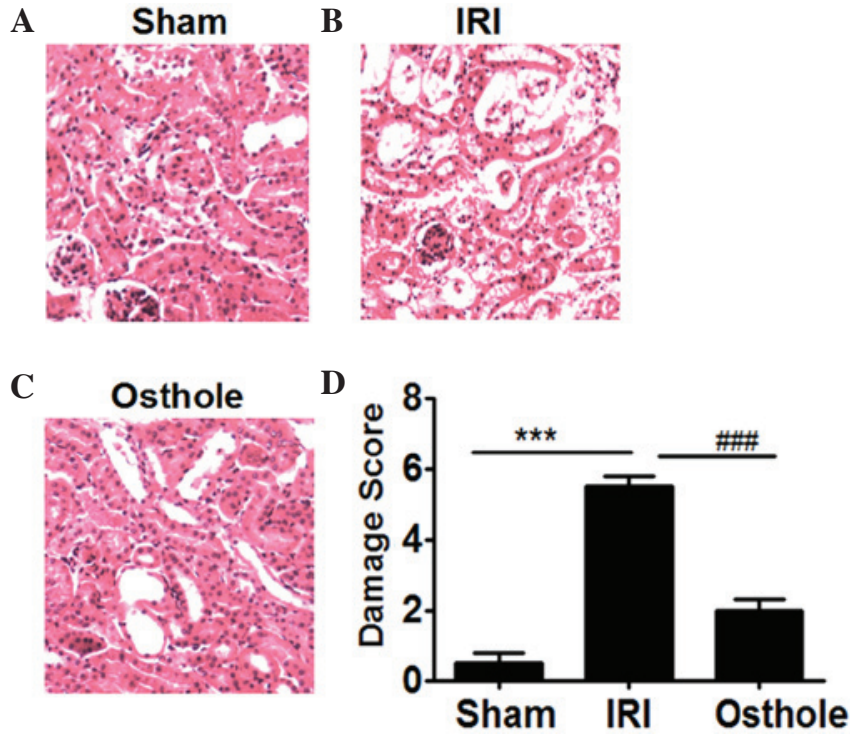

Figure 2. Hematoxylin and eosin staining for histopathological changes: Effects of osthole pretreatment on ischemia/reperfusion (I/R)-induced renal injury. (A) The Sham group shows no histopathological change $24 \mathrm{~h}$ after I/R injury (Sham; magnification, x200). (B) The I/R injury (IRI) group shows widespread degeneration of the tubular architecture, tubular dilation, tubular cell swelling, cellular vacuolization, tubular cell necrosis and inflammatory cell infiltration $24 \mathrm{~h}$ after IRI (magnification, x200). (C) The osthole group shows little degeneration of the tubular architecture, tubular dilation, tubular cell swelling, cellular vacuolization, tubular cell necrosis and inflammatory cell infiltration $24 \mathrm{~h}$ after IRI (magnification, x200). (D) Semi-quantitative assessment of the histological lesions based on renal histopathological changes. Data are presented as the mean \pm standard error of the mean $(n=10)$. ${ }^{* * * *} \mathrm{P}<0.001$, vs. Sham; ${ }^{\# \# \#} \mathrm{P}<0.001$, vs. osthole.

\section{Discussion}

The acute kidney injury induced by $\mathrm{I} / \mathrm{R}$ is a clinical and experimental syndrome characterized by renal dysfunction, extensive widespread tubular damage, tubular cell necrosis and inflammatory cell infiltration (4). Inflammatory responses are believed to play a central role in $\mathrm{I} / \mathrm{R}$ injury, in addition to several other factors such as apoptosis, necrosis and oxidative stress $(4,5)$. Inflammatory responses exert a range of deleterious effects on renal tissue, causing a cascade of injury that may lead to organ failure $(4,5)$.

The present results showed that sham operation did not alter the renal parameters (serum creatinine, BUN, histological features and inflammation) as compared with the IRI group rats. By contrast, renal $I / R$ worsened the renal dysfunction and histopathological features in rats. In the present study, the inflammatory response in the kidney during $\mathrm{I} / \mathrm{R}$ was evaluated by measuring the expression of proinflammatory cytokine TNF- $\alpha$, IL-6 and IL-8. In accordance with the change of renal parameters, the expression of proinflammatory cytokine TNF- $\alpha$, IL- 6 and IL- 8 were significantly increased due to I/R injury (13). Furthermore, histopathological alterations were evident in the ischemic rat kidney, as well as alteration of renal function and inflammation. The kidney of the rats in the IRI group that underwent 45 min ischemia followed by $24 \mathrm{~h}$ reperfusion showed a significant pathological difference, as manifested by widespread degeneration of tubular architecture, tubular dilation, tubular cell swelling, cellular vacuolization, tubular cell necrosis and inflammatory cell 
A

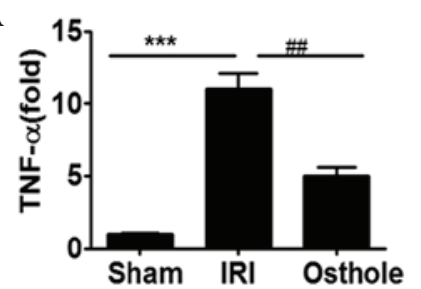

B

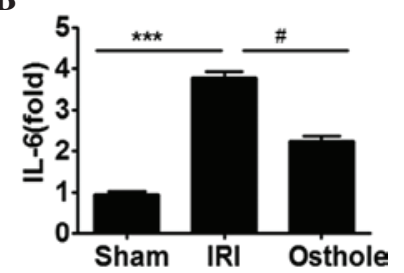

C

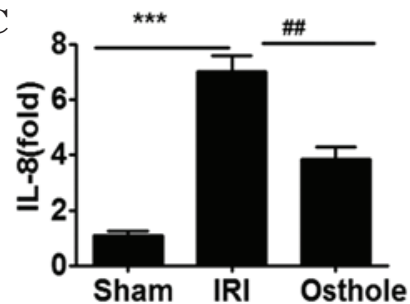

Figure 3. Effects of osthole pretreatment on the expression of proinflammatory cytokines after renal ischemia/reperfusion injury. Quantitative polymerase chain reaction was used to assess the expression of inflammatory cytokines in the kidney. The mRNA expression levels for (A) TNF- $\alpha$, (B) IL-6 and (C) IL-8 after IRI were selectively evaluated. Data are presented as the mean \pm standard error of the mean $(\mathrm{n}=10) .{ }^{* * * *} \mathrm{P}<0.001$ vs. sham; ${ }^{\# \#} \mathrm{P}<0.001$, ${ }^{\#} \mathrm{P}<0.05$ vs. osthole. TNF- $\alpha$, tumor necrosis factor- $\alpha$; IRI, ischemia/reperfusion vehicle group; IL-6, interleukin-6; IL-8, interleukin-8.

A

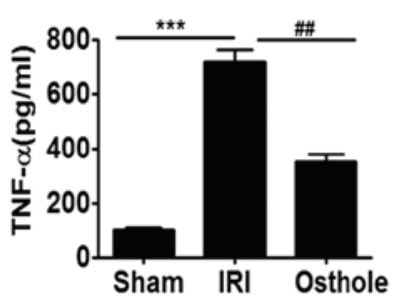

B

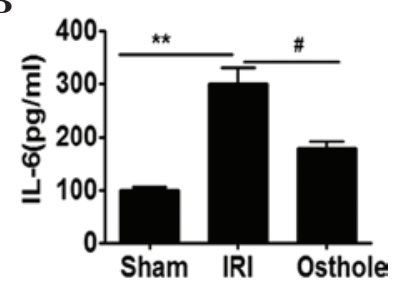

C

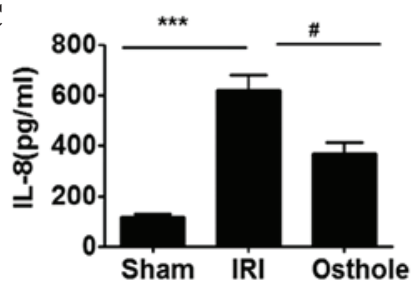

Figure 4. Effects of osthole pretreatment on the secretion of proinflammatory cytokine after renal ischemia/reperfusion injury. ELISA was employed to assess the expression of proinflammatory cytokine in the serum. The expression levels of (A) TNF- $\alpha$, (B) IL-6 and (C) IL- 8 after IRI were selectively detected. Data are presented as the mean \pm standard error of the mean $(n=10)$. ${ }^{* * * *} \mathrm{P}<0.001$ vs. sham; ${ }^{\# \#} \mathrm{P}<0.01,{ }^{\#} \mathrm{P}<0.05$ vs. osthole. TNF- $\alpha$, tumor necrosis factor- $\alpha$; IRI, ischemia/reperfusion vehicle group; IL-6, interleukin-6; IL-8, interleukin-8.

infiltration. Therefore, suppressing inflammation is a potential therapeutic target for reducing renal IRI.

Osthole is a Chinese herbal medicine that has been shown to have a widespread anti-inflammatory effect and can decrease cerebral I/R injury $(7,8)$. However, to date there is a lack of study regarding the precise function and potential mechanisms of osthole in renal I/R injury. The present results suggest that osthole protected the rats against renal $\mathrm{I} / \mathrm{R}$ injury as manifested by the attenuation of renal dysfunction, the histopathology alteration and the expression of the

A

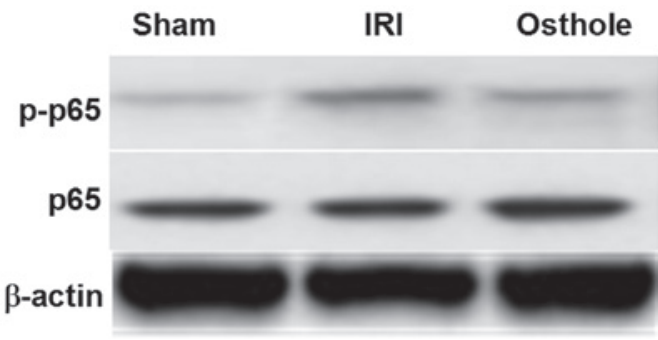

B

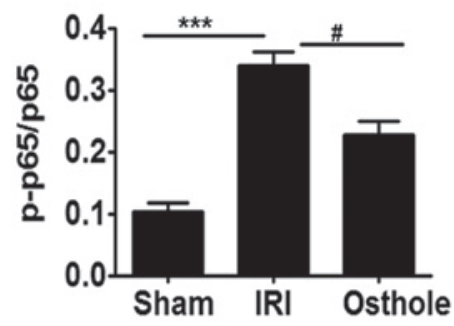

Figure 5. Effects of osthole pretreatment on the expression of p65 following renal ischemia/reperfusion injury. Western blot analysis was employed to evaluate the protein expression of p65 and p-p65. (A) Representative western blot analysis of p65. (B) Semi-quantitative analysis of 10 animals studied in each group. Relative quantities of p-p65 and p65 in each group of rats were normalized against $\beta$-actin and presented as a ratio between p-p65 and p65. ${ }^{* * * *} \mathrm{P}<0.001$ vs. sham; ${ }^{*} \mathrm{P}<0.05$ vs. IRI. IRI, ischemia/reperfusion vehicle group.

A

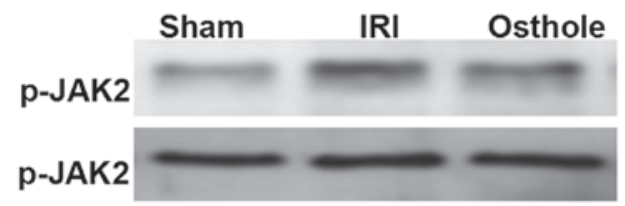

B

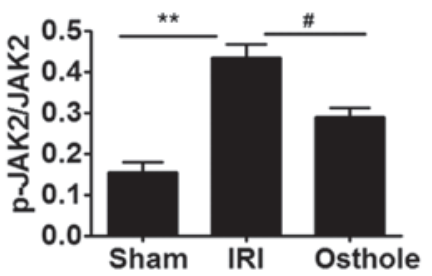

C

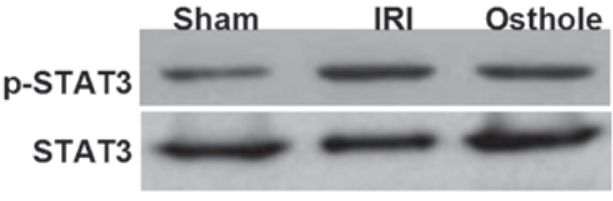

D

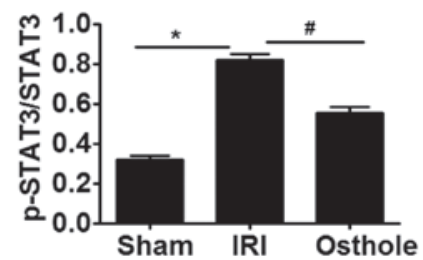

Figure 6. Effects of osthole pretreatment on the JAK2/STAT3 signaling after renal ischemia/reperfusion injury. Western blot analysis was employed to semi-quantitatively analyze the protein expression levels of JAK2, p-JAK2, STAT3 and p-STATA3. (A) Representative result for western blot analysis of JAK2. (B) Relative quantities of p-JAK2 and JAK2 in each group of rats were normalized against $\beta$-actin and presented as a ratio between p-JAK2 and JAK2. ${ }^{* *} \mathrm{P}<0.01$ vs. sham; ${ }^{*} \mathrm{P}<0.05$ vs. IRI. (C) Representative result for western blot analysis of STAT3. (D) Relative quantities of STAT3 and p-STAT3 in each group of rats were normalized against $\beta$-actin and presented as a ratio between p-STAT3 and STAT3. ${ }^{*} \mathrm{P}<0.05$ vs. sham; ${ }^{\#} \mathrm{P}<0.05$ vs. IRI. 
proinflammatory cytokines TNF- $\alpha$, IL- 6 and IL-8 induced by renal I/R injury.

$\mathrm{NF}-\kappa \mathrm{B}$ is a ubiquitously acting transcription factor associated with immune and inflammatory reactions (5). NF- $\mathrm{KB}$ has been shown to regulate the expression of the proinflammatory cytokines TNF- $\alpha$, IL- 6 and IL- 8 , which contribute to the further amplification of inflammation $(5,14)$. Furthermore, NF- $\kappa \mathrm{B}$ is crucial to the propagation of the inflammatory response in the renal I/R injury and its activation is primarily dependent $\mathrm{p} 65$ activation $(4,5)$. In the present study, I/R insult or osthole influenced the expression of p65; however, the expression of p-p65 was significantly increased as compared with the sham group. In addition, osthole pretreatment can decrease the expression of p-p65 in IRI, which indicated Osthole pretreatment may significantly reduce $\mathrm{NF}-\kappa \mathrm{B}$ activation.

The JAK2/STAT3 pathway is a classical signaling which has been demonstrated to regulate inflammation associated with renal I/R injury (6). Therefore, the effect of osthole on JAK2/STAT3 signaling were investigated in a rat model of I/R. I/R injury and osthole appear to increase JAK2 activation via phosphorylation, as manifested by the increased levels of p-JAK2 detected in the IRI group rats compared with the sham group rats. Furthermore, preconditioning of rats with osthole can significantly suppress JAK2 activation, as manifested by the reduced levels of p-JAK2 in osthole group rats compared with IRI group rats. This result lead us to evaluate STAT3 activity, as JAK2 activation is known to induce STAT3 activation. Consistent with the aforementioned results, I/R injury and osthole influence the expression of STAT3. However, I/R injury can increase the expression of p-STAT3 and osthole pretreatment can reduce the expression of p-STAT3 induced by I/R injury. These results indicated that IRI can induce the activation of STAT3, and osthole pretreatment can attenuate the activation of p-STAT3 induced by I/R injury. Previous results suggest that blocking JAK2/STAT3 activation can decrease I/R-induced renal injury (6); therefore, in the current report we did not conduct additional studies to demonstrate that suppressing JAK2/STAT3 signaling can decrease I/R-induced NF- $\mathrm{B}$ activation in the kidney. Considering the capacity of osthole preconditioning to prevent I/R-induced renal injury, it is notable that osthole could regulate additional pathways other than the JAK2/STAT3 pathway to suppress NF- $\kappa$ B activation, such as the MAPK kinase cascade (15). Therefore, further studies are required to investigate the pathways involved in the suppression of NF- $\kappa \mathrm{B}$ activation by osthole in the context of I/R injury.

In summary, the present results suggest that precondition rats with osthole attenuated renal I/R injury, as manifested by the reduction of pathological and serum changes. Mechanistic studies demonstrated that osthole preconditioning is able to decrease $N F-\kappa B$ activation by suppressing the activation of JAK2/STAT3. Collectively, these data support the conclusion that osthole may offer an alternative therapy for the prevention of renal I/R injury in the clinical practice.

\section{Acknowledgements}

This study was supported by the Sichuan Provincial Natural Science Foundation (grant no. 130501).

\section{References}

1. Mangano CM, Diamondstone LS, Ramsay JG, Aggarwal A, Herskowitz A and Mangano DT: Renal dysfunction after myocardial revascularization: Risk factors, adverse outcomes and hospital resource utilization. The multicenter study of perioperative ischemia research group. Ann Intern Med 128: 194-203, 1998.

2. Schiffl H, Lang SM and Fischer R: Daily hemodialysis and the outcome of acute renal failure. N Engl J Med 346: 305-310, 2002.

3. Chertow GM, Burdick E, Honour M, Bonventre JV and Bates DW: Acute kidney injury, mortality, length of stay and costs in hospitalized patients. J Am Soc Nephrol 16: 3365-3370, 2005.

4. Lau A, Wang S, Liu W, Haig A, Zhang ZX and Jevnikar AM: Glycyrrhizic acid ameliorates HMGB1-mediated cell death and inflammation after renal ischemia reperfusion injury. Am J Nephrol 40: 84-95, 2014.

5. Ranganathan PV, Jayakumar C, Mohamed R, Dong Z and Ramesh G: Netrin-1 regulates the inflammatory response of neutrophils and macrophages and suppresses ischemic acute kidney injury by inhibiting COX-2-mediated PGE2 production. Kidney Int 83: 1087-1098, 2013.

6. Si YN, Bao HG, Xu L, Wang XL, Shen Y, Wang JS and Yang XB: Dexmedetomidine protects against ischemia/reperfusion injury in rat kidney. Eur Rev Med Pharmacol Sci 18: 1843-1851, 2014.

7. Liu J, Zhang W, Zhou L, Wang X and Lian Q: Anti-inflammatory effect and mechanism of osthole in rats. Zhong Yao Cai 28: 1002-1006, 2005.

8. Li F, Gong Q, Wang L and Shi J: Osthole attenuates focal inflammatory reaction following permanent middle cerebral artery occlusion in rats. Biol Pharm Bull 35: 1686-1690, 2012.

9. Zheng Y, Lu M, Ma L, Zhang S, Qiu M and Ma X: Osthole ameliorates renal ischemia-reperfusion injury by inhibiting inflammatory response. Urol Int 91: 350-356, 2013.

10. Fang J, He L, Wang SQ, Ma MJ, Liu HY, Zhu XH, Zhu P, Wei X and Wang CY: A simplified two-stitch sleeve technique for arterial anastomosis of cervical heterotopic cardiac transplantation in mice. Am J Transl Res 5: 521-529, 2013.

11. Zheng X, Feng B, Chen G, Zhang X,Li M, Sun H, Liu W, Vladau C, Liu R, Jevnikar AM, et al: Preventing renal ischemia-reperfusion injury using small interfering RNA by targeting complement 3 gene. Am J Transplant 6: 2099-2108, 2006.

12. Zhang S, Lv JW, Yang P, Yu Q, Pang J, Wang Z, Guo H, Liu S, Hu J, Li J, et al: Loss of dicer exacerbates cyclophosphamide-induced bladder overactivity by enhancing purinergic signaling. Am J Pathol 181: 937-946, 2012.

13. Yang S, Chou WP and Pei L: Effects of propofol on renal ischemia/reperfusion injury in rats. Exp Ther Med 6: 1177-1183, 2013.

14. Wang $\mathrm{X}$, Xiong $\mathrm{M}$, Zeng $\mathrm{Y}$, Sun $\mathrm{X}$, Gong $\mathrm{T}$ and Zhang Z: Mechanistic studies of a novel mycophenolic acid-glucosamine conjugate that attenuates renal ischemia/reperfusion injury in rat. Mol Pharm 11: 3503-3514, 2014.

15. Hao JL, Li YF and Li RS: A novel mechanism of NALP3 inducing ischemia reperfusion injury by activating MAPK pathway in acute renal failure. Med Hypotheses 80: 463-465, 2013. 